\title{
Fabrication and Characterization of Gliclazide Loaded Microcapsules
}

\author{
Muhammad Asad $^{1,2}$, Sajid Bashir ${ }^{1}$, TariqMahmood ${ }^{2 *}$, Imran Nazir $^{1,3}$, Muhammad Imran ${ }^{1}$, \\ Sabiha Karim ${ }^{4}$ and Fakhar ul Hassnain ${ }^{1}$ \\ ${ }^{1}$ Faculty of Pharmacy; University of Sargodha; Sargodha - Pakistan. ${ }^{2}$ Faculty of Pharmacy; University of Central \\ Punjab; Lahore - Pakistan. ${ }^{3}$ Department of Pharmacy; COMSATS Institute of Information Technology; Abbottabad \\ - Pakistan. ${ }^{4}$ College of Pharmacy; University of Punjab; Lahore - Pakistan
}

\begin{abstract}
This study aimed to formulate, characterize and evaluate the Gliclazide (GLZ) microcapsules prepared with sodium alginate, guar gum and pectin in different ratios by ionotropic-gelation method. The microcapsules were evaluated against different parameters such as particle size and shape, Carr's index, Hausner's ratio, rheological studies and drug release kinetics. Fourier Transform Infra Red (FTIR) and Differential Scanning Calorimetric (DSC) studies demonstrated the absence of any drug - polymers interaction. Promising characteristics were observed in rheological behavior and release kinetics. The size of microcapsules and percentage yield was in the range of 676 to $727 \mu \mathrm{m}$ and 69 to 77\%, respectively. Scanning electron micrographs revealed that microcapsules were discrete, spherical and free flowing. Entrapment efficiency and uniform drug release kinetics were some of the probable characteristics depicting the novel formulation design of Gliclazide microcapsules.
\end{abstract}

Key words: Microcapsules; gliclazide; ionotropic-gelation method; guar gum; pectin

\section{INTRODUCTION}

Microencapsulation is a process designed for the sustained release of drugs. For sustained action of drugs, polymeric microcapsules are among the options to deliver the drug at the target site. In these systems, active ingredient is enclosed in a coat of polymer, thus one can expect advantages such as decreased dosing frequency, increased oral bioavailability, decreased side effects and ultimately patient compliance (Getsios et al. 2004). Microparticles offer various significant advantages as drug delivery systems, including: (i) an effective protection of the encapsulated active agent against (e.g. enzymatic) degradation, (ii) the possibility to accurately control the release rate of the incorporated drug over the periods of hours to months, (iii) an easy administration (compared to alternative parenteral controlled release dosage forms, such as macro-sized implants), and (iv) desired, pre-programmed drug release profiles can be provided, which match the therapeutic needs of the patient. Major mechanisms of drug release from the microcapsules include diffusion, dissolution, osmosis and erosion (Singh et al. 2010).

Current study can better offer for the patients suffering from long term non-insulin dependent diabetes mellitus (NIDDM). Diabetes mellitus is a group of syndrome characterized by hyperglycemia, altered metabolism of lipids, carbohydrates, proteins and an increased risk of complication from the vascular diseases (Nerup et al. 1974). Through microencapsulation of GLZ,

*Author for correspondence: tariqmahmood750@gmail.com 
one could achieve the goal of control release and to determine drug release parameters in accordance with different release kinetic models (Yamuda et al. 2001; Mankala et al. 2010)

GLZ (Fig. 1) is a second-generation sulfonylurea oral hypoglycemic agent that is preferred therapy for NIDDM, due to its selective inhibitory action towards pancreatic $\mathrm{K}^{+}$ATP channels (Kobayashi et al. 1984). It is necessary to select a formulation, which can bypass stomach as majority of Gliclazide is absorbed in duodenum and jejunum, while some portion of drug is absorbed in mid and distal GI regions (Grbic et al. 2011).

This study aimed to formulate, characterize and evaluate the Gliclazide (GLZ) microcapsules prepared with sodium alginate, guar gum and pectin in different ratios by ionotropic-gelation method.<smiles>Cc1ccc(S(=O)(=O)NC(=O)NN2CC3CCCC3C2)cc1</smiles>

Figure 1 - Chemical structure of gliclazide.

\section{MATERIAL AND METHODS}

\section{Materials}

Gliclazide was gifted by the Stand Pharma Lahore, Pakistan. Sodium alginate, pectin, and guar gum were purchased from China, BDH Laboratories and Merck, respectively. Sodium hydroxide and calcium chloride was also purchased from Merk. Mono basic potassium phosphate and chloroform were purchased from Riedel de Haen; all other chemicals and solvents used were of analytical grade.

\section{Preparation of gliclazide loaded microcapsules} Gliclazide loaded microcapsules of calcium alginate were prepared by the ionotropic gelation method (George and Abraham 2006). In this method, GLZ microcapsules were prepared by using sodium alginate in combination with different ratios of polymers; pectin and guar gum as shown in Table 1 . Sodium alginate $(1.75 \mathrm{~g})$ and different ratios of polymers were dissolved in distilled water $(100 \mathrm{~mL})$ to form a homogeneous solution using magnetic stirrer. GLZ (2.0 g) was dissolved in $100 \mathrm{~mL}$ of chloroform. The solution of drug and polymer was mixed by magnetic stirrer at speed of $1000 \mathrm{rpm}$ to form homogeneous solution. Then drug - polymer solution was added drop wise to a $\mathrm{CaCl}_{2}(25 \mathrm{w} / \mathrm{v})$ solution $(100 \mathrm{~mL})$ by using hypodermic syringe (needle size $26 \mathrm{G}$ ). The droplets were allowed to harden in gelling bath for $30 \mathrm{~min}$ to produce the spherical rigid microcapsules, then filtered, washed repeatedly with distilled water and dried in oven at $37^{\circ} \mathrm{C}$ until a constant weight was obtained.

Table 1 - Composition of various formulations of Gliclazide microcapsules.

\begin{tabular}{ccccc}
\hline $\begin{array}{c}\text { Formulation } \\
\text { code }\end{array}$ & $\begin{array}{c}\text { Gliclazide } \\
(\% \mathbf{w} / \mathbf{v})\end{array}$ & $\begin{array}{c}\text { Sodium } \\
\text { alginate } \\
(\boldsymbol{\%} \mathbf{w} / \mathbf{v})\end{array}$ & $\begin{array}{c}\text { Pectin } \\
(\boldsymbol{\%} / \mathbf{v})\end{array}$ & $\begin{array}{c}\text { Guar } \\
\text { Gum } \\
(\% \mathbf{\%})\end{array}$ \\
\hline GLZ 1 & 2 & 1.75 & - & - \\
GLZ 2 & 2 & 1.75 & 0.5 & - \\
GLZ 3 & 2 & 1.75 & 0.75 & - \\
GLZ 4 & 2 & 1.75 & 1 & - \\
GLZ 5 & 2 & 1.75 & - & 0.5 \\
GLZ 6 & 2 & 1.75 & - & 0.75 \\
GLZ 7 & 2 & 1.75 & - & 1 \\
GLZ 8 & 2 & 1.75 & 1 & 0.5 \\
\hline
\end{tabular}

\section{Characterization and evaluation of microsphere Percentage yield}

The percentage yield of microcapsules produced was calculated using eq 1 (Ziyaur et al. 2006).

Percentage yield $=$ Practical weight [microcapsules] $\times 100$

Theoretical weight [drug and polymer]

\section{Particle size analysis}

All the formulations prepared were analyzed for the particle size by placing the microcapsules on a set of standard sieves ranging from sieve number 10-40. The sieve was arranged in descending order with mesh size number 10 on the top and number 40 at the bottom. The microcapsules were introduced from the top and after completion of shaking, the weight of microcapsules that was retained was determined. Then the average mean diameter was calculated and considered as mean particle size and determined by eq (2).

\section{Mean particle size $=$ \\ $\Sigma$ [mean particle size of fraction $\mathrm{x}$ weight fraction] $\Sigma$ [weight fraction]}

\section{Assessment of micromeritic properties}

The microcapsules were characterized by various micromeritic properties. The angle of repose of the 
microcapsules, which measured the resistance to particle flow, was determined by the fixed funnel method and calculated as in eq (3).

$\theta=\tan ^{-1} \mathrm{~h} / \mathrm{r}$

Where ' $h$ ' is height and ' $r$ ' is radius of the heap. The angle of repose less than 30 shows excellent flow properties. The Carr's index was evaluated for the flow ability of the powder by comparing the pour density and tapped density of microcapsules and was calculated by using eq (4). Carr's index $=\{(\rho t-\rho b) \times 100\} / \rho t$

Where $\rho b$ is bulk density and $\rho t$ is tapped density. This was measured in a $10 \mathrm{~mL}$ graduated cylinder. Carr's index less than $15 \%$ gives good flow characteristics and above $25 \%$ indicates poor flow characteristics (Aulton 1990). Hausner's ratio, another index of flowability, was measured by eq (5).

Hausner's ratio $=\rho t / \rho b$

Hausner's ratio value between 1.00-1.11 show excellent flow ability.

\section{Flow properties of the microcapsules}

Angle of repose

A funnel was fixed in a stand at specific height from the surface of the graph paper. Then the weighed amount of microcapsules was passed through the funnel so that they formed a static heap. The height [h] and radius [r] of the heap was measured and angle of repose was calculated by using the formula.

$\theta=\tan ^{-1} \mathrm{~h} / \mathrm{r}$

\section{Bulk density}

Bulk density of microcapsules was determined by using the following formula (Banker et al. 1987).

Bulk density $\left[\rho_{b}\right]=\underline{\text { Weight of microcapsules }}$ Bulk volume

\section{Tapped density}

The tapped density was measured by employing the conventional tapping method using $10 \mathrm{~mL}$ measuring cylinder and the number of tapings were 100 as it was sufficient to bring about a plateau condition. Tapped density was calculated by the following formula (Shariff A et al. 2007).

Tapped density $=$ Weight of microcapsules

Volume of microcapsules after 100 tapings

Carr's index

Carr's index of microcapsules was calculated by the following equation.
Carr's index $=$ Tapped density - bulk density $\mathrm{x} 100$

$$
\text { Tapped density }
$$

Tapped density and bulk density were measured by the cylinder. Carr's value less than $10 \%$ shows excellent flow properties.

\section{Hausner's ratio}

Hausner's ratio is used to determine the flow properties and can be calculated as described by Shariff et al. (2007).

Hausner's ratio $=\rho t / \rho_{b}$

Where $\rho t$ is tapped density and $\rho b$ is bulk density. The value of Hausner's ratio in the range of 1.001.11 show excellent flow properties.

\section{Percentage Encapsulation Efficiency}

The encapsulation efficiency of microcapsules was determined by crushing $100 \mathrm{mg}$ of dried microcapsules using pestle and mortar. Then the ground microcapsules were placed in $100 \mathrm{~mL}$ of phosphate buffer prepared with USP specifications with $\mathrm{pH} 7.4$ and vortexing carried for $5 \mathrm{~min}$. The sample was then filtered with Whatmann filter paper number 4 to obtain clear solution. The filtered sample was suitably diluted and analyzed for drug content in spectrophotometer (Pharmaspec 1700, Shimadzu, Japan) at $226 \mathrm{~nm}$ (Vijaya et al. 2006). The absorbance measured was then converted into the amount of GLZ by using the standard calibration curve. The percentage entrapment efficiency was calculated by using the formula mentioned in eq 6 .

Encapsulation efficiency $=$

Estimated percentage drug content x 100

Theoretical percentage drug content

\section{Shape and surface analysis}

The surface morphology of the microcapsules was analyzed by scanning electron microscopy (Model S3400N). The dried microcapsules were sprinkled on the adhesive tape, which was then smacked to gold coated (thickness $150-200^{\circ} \mathrm{A}$ ) aluminum end. The pictures of microcapsules were taken by random scanning of the aluminum end under reduced pressure.

\section{Fourier transform infrared (ftir) studies}

FTIR spectrum is one of the important analytical techniques to determine the structure of the compound and predict the drug-polymer interaction. In this method the individual microcapsules were crushed in a pestle and mortar. 
The crushed material (1.0 mg) was mixed with 100 $\mathrm{mg} \mathrm{KBr}$, which was then subjected to compression pressure of 10 tons for 2 min to prepare the disc. Then FTIR spectra of the scanning wavelength range $4000-400 \mathrm{~cm}^{-1}$ were recorded by using an FTIR spectrophotometer (IR-Prestige-21, Shimadzu, Japan).

\section{Differential scanning calorimetric (DSC) analysis}

DSC analysis was done to characterize the changes observed during the preparation of microcapsules and was carried out by using Thermal analyser (SDT Q 600, Shimadzu, Japan). Gliclazide, polymers and drug-loaded microcapsules were triturated separately and heated in sealed aluminium pans at a rate of $10^{\circ} \mathrm{C} / \mathrm{min}$ from 30 to $400^{\circ} \mathrm{C}$ temperature range under a nitrogen flow of $40 \mathrm{~mL} / \mathrm{min}$ (Tayade et al. 2004).

\section{In vitro drug release studies}

In Vitro drug release studies of Gliclazide microcapsules were carried out by using the USP dissolution rate test apparatus type II (Pharmatest) in $900 \mathrm{~mL} 0.1 \mathrm{~N} \mathrm{HCl}$ for the first $2 \mathrm{~h}$ and then in phosphate buffer $\left(\mathrm{pH} \mathrm{7.4)}\right.$ at $37^{\circ} \mathrm{C} \pm 0.5 \mathrm{C}$ for $8 \mathrm{~h}$ at $100 \mathrm{rpm}$. Microcapsules were enclosed in hard gelatin capsules in a quantity equivalent to $120 \mathrm{mg}$ dose of the drug. The capsules were then placed into the dissolution medium. The samples of dissolution medium $(5.0 \mathrm{~mL})$ was withdrawn at definite intervals and filtered through a Whatmann filter paper. The equivalent volume of the medium with the same temperature was added into the dissolution vessel. For Gliclazide contents, the sample was analyzed at a wavelength of $226 \mathrm{~nm}$ after suitable dilution.

The release kinetics of Gliclazide from the microcapsules was also evaluated by using various release kinetic models, i.e Zero order, First order, Higuchi model, Hixson Crowell model and Korsmeyer peppas model (Ranjha et al. 2010).

\section{RESULTS AND DISCUSSION}

\section{Flow behavior of microcapsules}

Gliclazide microcapsules were developed by the ionotropic gelation method, employing pectin and guar gum as polymers. The mean particle size of microcapsules significantly increased with increasing polymer concentration. Increase in the coating layer or polymer ratio around the drug proportionally increased the thickness of microcapsules and vice versa. The particle size of the microcapsules was in the range of $676 \pm 1.73$ to $727 \pm 1.73 \mu \mathrm{m}$, whereas the percentage yield of various formulations varied between $69.17 \pm 0.47$ to $77.47 \pm 0.21 \%$ as shown in Table 2 .

Table 2 - Physical and Rheological properties of microcapsules.

\begin{tabular}{ccccccc}
\hline Formulation & $\begin{array}{c}\text { Mean particle } \\
\text { size }[\mu \mathrm{m}]\end{array}$ & $\begin{array}{c}\text { \% Age Yield } \\
{[\%]}\end{array}$ & $\begin{array}{c}\text { Angle of } \\
\text { Repose }\end{array}$ & $\begin{array}{c}\text { Carr's } \\
\text { Index }\end{array}$ & $\begin{array}{c}\text { Hausner' } \\
\text { s Ratio }\end{array}$ & $\begin{array}{c}\text { \% Age } \\
\text { Entrapment }\end{array}$ \\
\hline GLZ 1 & $676 \pm 1.73$ & $71.73 \pm 0.53$ & $26.54 \pm 0.20$ & $7.33 \pm 0.38$ & 1.07 & $59.92 \pm 0.36$ \\
GLZ 2 & $689 \pm 1.0$ & $69.17 \pm 0.47$ & $26.38 \pm 0.34$ & $9.05 \pm 0.91$ & 1.10 & $62.85 \pm 0.41$ \\
GLZ 3 & $696 \pm 2.64$ & $70.22 \pm 0.22$ & $25.72 \pm 0.01$ & $6.86 \pm 0.58$ & 1.07 & $63.68 \pm 0.20$ \\
GLZ 4 & $705 \pm 1.73$ & $73.47 \pm 0.21$ & $25.15 \pm 0.47$ & $6.39 \pm 0.29$ & 1.06 & $65.35 \pm 0.20$ \\
GLZ 5 & $697 \pm 2.0$ & $75.05 \pm 0.47$ & $24.24 \pm 0.21$ & $4.75 \pm 0.90$ & 1.04 & $72.24 \pm 0.75$ \\
GLZ 6 & $710 \pm 2.64$ & $76.66 \pm 0.44$ & $23.97 \pm 0.01$ & $5.99 \pm 0.72$ & 1.06 & $73.91 \pm 0.55$ \\
GLZ 7 & $718 \pm 2.64$ & $77.47 \pm 0.21$ & $23.46 \pm 0.12$ & $8.24 \pm 0.61$ & 1.09 & $75.17 \pm 0.36$ \\
GLZ 8 & $727 \pm 1.73$ & $73.52 \pm 0.57$ & $25.55 \pm 0.37$ & $7.56 \pm 0.96$ & 1.08 & $78.09 \pm 0.55$ \\
\hline
\end{tabular}

Packing property of the pure drug and microcapsules was studied with the help of loose bulk density and tapped bulk density. As shown by the results, the value of loose bulk density was lower as compared to the tapped bulk density because the active ingredient was more bulky as well as fluffy in nature that indicates more interparticulate space between the pure drug than microcapsules resulting excellent flow behavior of Gliclazide microcapsules. Compressibility of the drug and microcapsules were studied by using Carr's index, angle of repose and Hausner's Ratio. Pure drug showed poor flow and compressibility values, which might be due to its bulky nature while Gliclazide microcapsules showed excellent flow behavior. The angle of repose for microcapsules was between 23.4 to $26.5^{\circ}$, indicating good flow property. The findings were further supported by Carr's index, which was below $10 \%$ and Hausner's ratio, which was less 
than 1.11. Among all the formulations, GLZ-5 showed good micromeritic properties.

\section{Scanning Electron Microscopy (SEM)}

Scanning electron microscopy of Gliclazide loaded microcapsules (Fig. 2) revealed that microcapsules prepared with varying polymers showed nearly spherical shape. Some cracks were present in GLZ 1 and GLZ 8 that indicated poor entrapment of the drug by the polymer ratio used in these formulations. But formulation GLZ-5 showed good entrapment of the drug with nearly spherical shape and also had good release characteristic, which was confirmed by in-vitro drug release studies. SEM photographs also revealed the absence of crystals of drug on the surface of the microcapsules made with polymers indicating uniform distribution of the drug within these microcapsules.

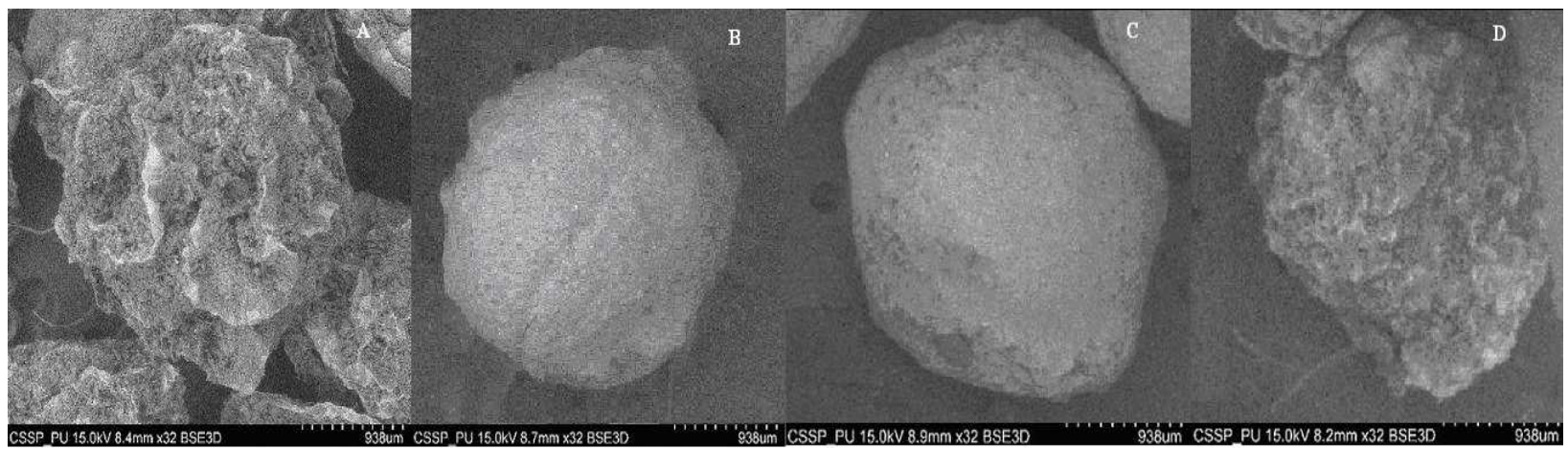

Figure 2 - Scanning electron micrographs of Gliclazide loaded microcapsules A: GLZ-1, B: GLZ-2, C: GLZ-5, D: GLZ-8.

Fourier transform infrared spectroscopy (FTIR)

FTIR spectra of pure drug and polymers were obtained (Fig. 3) and analyzed for characteristic peaks. Peaks at $1444 \mathrm{~cm}^{-1}$ and $1583 \mathrm{~cm}^{-1}$ indicated $\mathrm{C}=\mathrm{C}$ stretching of benzyl ring; $\mathrm{C}=\mathrm{O}$ stretching was observed through the peaks at $1159 \mathrm{~cm}^{-1}$ and 1710 $\mathrm{cm}^{-1}$; C-H asymmetric stretching at peaks at 2310 $\mathrm{cm}^{-1}$ and symmetric stretching at $2850 \mathrm{~cm}^{-1}$; peak at $3265 \mathrm{~cm}^{-1}$ indicated $\mathrm{N}-\mathrm{N}$ stretching. All these principle peaks indicated that the drug was finely dispersed in the polymers and there was no marked interaction between them. No new bands were detected in the spectra and drug was chemically stable even after encapsulation, which proved that polymers used in this formulation had no interaction with the drug.

\section{Differential Scanning Calorimeteric Analysis [DSC]}

Interaction of drug and polymer was further confirmed with the help of DSC analysis to observe the melting activities of the drug and polymers. Figure 4 illustrates the DSC thermogram of Gliclazide, sodium alginate, pectin, guar gum and Gliclazide loaded microcapsules [GLZ 1, GLZ2, GLZ5 and GLZ8]. A sharp endothermic peak corresponding to the melting point of Gliclazide was observed at $163-169^{\circ} \mathrm{C}$.

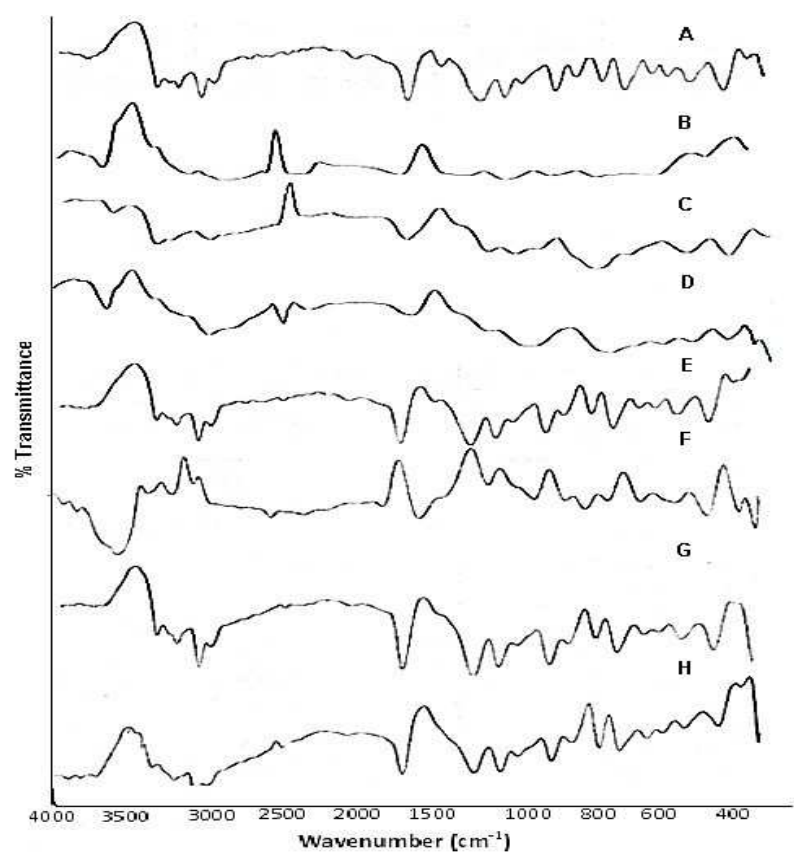

Figure 3 - FTIR Spectra of components and microcapsules where A: Gliclazide B: Sodium Alginate C: Pectin D: Guar Gum E: GLZ-1 F: GLZ-2 G: GLZ-5 H: GLZ-8. 
In case of sodium alginate, pectin and guar gum, exothermic peak was observed in the temperature range of $>300,155$ and $93^{\circ} \mathrm{C}$, respectively. In microcapsules characteristics, well recognizable thermal profile of the drug at the temperature corresponding to its melting point was also observed, which indicated the absence of any drug-polymer interaction (Logannathan et al. 2003). The results of this study showed significant melting ranges of the drug and polymers.

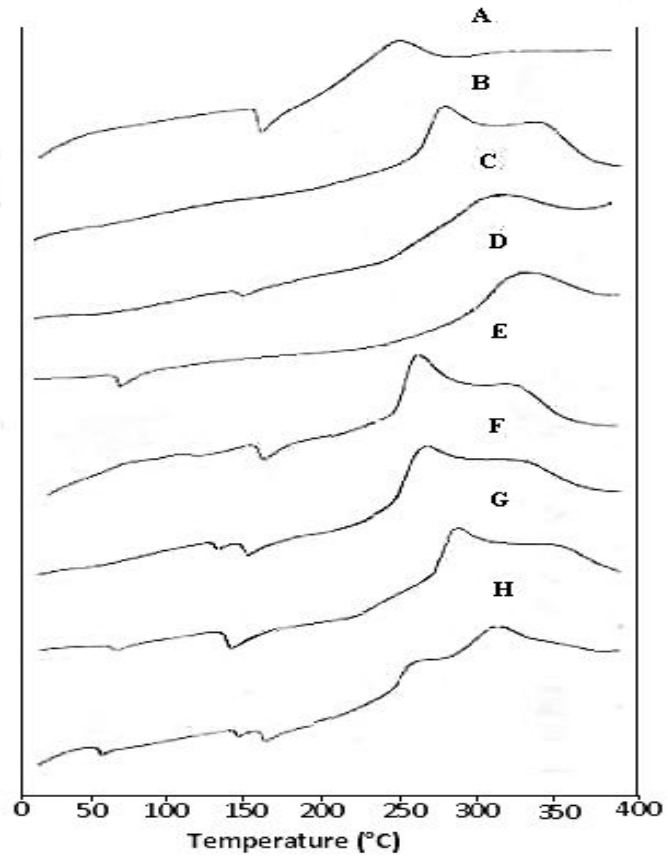

Figure 4 - DSC Thermo grams of components and microcapsules where A: Gliclazide B: Sodium Alginate C: Pectin D: Guar Gum E: GLZ-1 F: GLZ-2 G: GLZ-5 H: GLZ-8.

\section{In vitro drug release studies}

In vitro release studies of microcapsules were carried out in order to study the drug release behavior of the polymer matrix. The release was studied in $0.1 \mathrm{~N} \mathrm{HCl}$ and phosphate buffer $(\mathrm{pH} \mathrm{7.4)}$ for $12 \mathrm{~h}$ using USP type II dissolution apparatus. Data indicated that increase in the drug polymer ratio, correspondingly decreased the drug release from microcapsules as shown in Figure 5. It was observed that Gliclazide released slowly from the microcapsules, which was controlled over extended period and also that the release was dependent on the nature of the polymer used. The drug release from the microcapsules at $\mathrm{pH} 7.4$ was sustained over a prolonged period of time with greater retardation in microcapsules containing guar gum polymer. These microcapsules gave very slow release of the drug because of low permeability to water and this proved to be the best formulation.

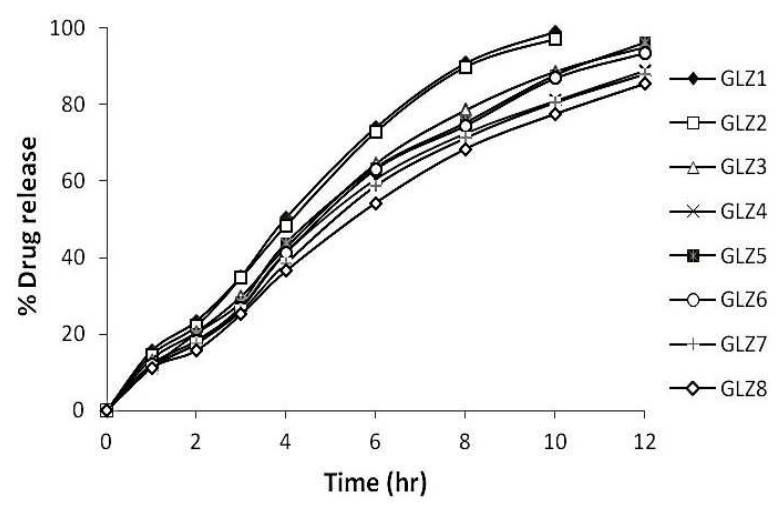

Figure 5 - In vitro drug release profile of various formulations of microcapsules.

\section{Kinetic release models}

Drug release kinetics of microcapsules depend upon the degree of gelation, hydration and erosion of the polymers. From the release kinetics, it was observed that the best fitting release kinetic was higuchi model with highest regression coefficient $\left[\mathrm{R}^{2}=0.995\right]$ as shown in Figure 6. Microcapsules showed non-fickian release by applying Korsmeyer-Peppas model [Power law] in which $n$ value ranged between 0.847 and 0.889 as described in Table 3. This indicated anomalous release mechanism marked by the diffusion as well as dissolution release mechanism. The Hixson Crowell model indicated the dissolution studies as a function of time. All these results were in accordance with the results found by microcapsules of flurbiprofen (Ranjha et al. 2010).

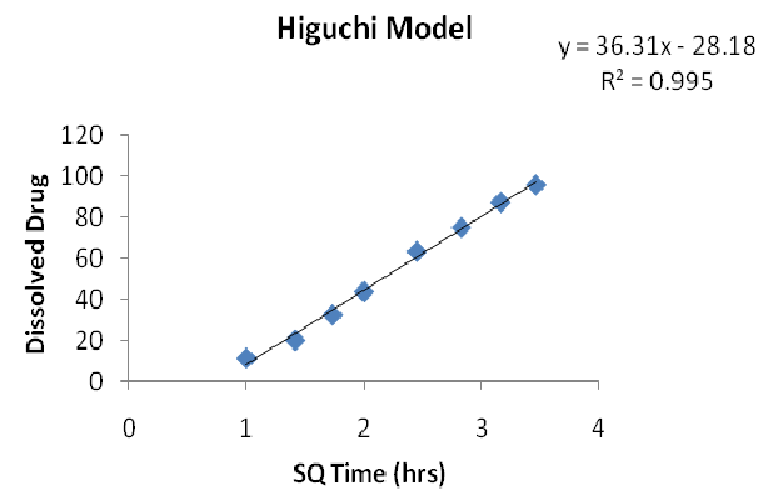

Figure 5 - Higuchi Kinetic model of GLZ 5. 
Table 3 - Release kinetic data of various formulations of microcapsules.

\begin{tabular}{ccccccccccccc}
\hline \multirow{2}{*}{ Formulation } & \multicolumn{2}{c}{ Zero order } & \multicolumn{2}{c}{ First order } & \multicolumn{2}{c}{ Higuchi } & \multicolumn{3}{c}{ Korsmeyer-Peppas } & \multicolumn{3}{c}{ Hixson-Crowell } \\
& $\mathrm{k}_{\mathrm{o}}$ & $\mathrm{R}^{2}$ & $\mathrm{~K}_{1}$ & $\mathrm{R}^{2}$ & $\mathrm{k}_{\mathrm{HC}}$ & $\mathrm{R}^{2}$ & $\mathrm{k}_{\mathrm{p}}$ & $\mathrm{N}$ & $\mathrm{R}^{2}$ & $\mathrm{k}_{\mathrm{H}}$ & $\mathrm{R}^{2}$ \\
\hline GLZ 1 & 9.936 & 0.976 & 0.47 & 0.885 & 42.29 & 0.983 & 0.144 & 0.863 & 0.986 & 0.377 & 0.984 \\
GLZ 2 & 9.876 & 0.974 & 0.376 & 0.945 & 42.06 & 0.982 & 0.135 & 0.889 & 0.987 & 0.383 & 0.982 \\
GLZ 3 & 7.916 & 0.968 & 0.258 & 0.972 & 36.40 & 0.985 & 0.127 & 0.847 & 0.987 & 0.333 & 0.971 \\
GLZ 4 & 7.406 & 0.964 & 0.191 & 0.990 & 34.12 & 0.986 & 0.110 & 0.879 & 0.983 & 0.333 & 0.967 \\
GLZ 5 & 7.819 & 0.975 & 0.264 & 0.942 & 36.31 & 0.995 & 0.116 & 0.889 & 0.990 & 0.340 & 0.969 \\
GLZ 6 & 7.897 & 0.969 & 0.236 & 0.974 & 36.25 & 0.984 & 0.112 & 0.889 & 0.984 & 0.343 & 0.971 \\
GLZ 7 & 7.404 & 0.970 & 0.186 & 0.990 & 34.02 & 0.986 & 0.105 & 0.888 & 0.987 & 0.335 & 0.971 \\
GLZ 8 & 7.176 & 0.977 & 0.167 & 0.990 & 32.86 & 0.986 & 0.100 & 0.889 & 0.984 & 0.332 & 0.976 \\
\hline
\end{tabular}

\section{CONCLUSION}

Gliclazide microcapsules were prepared successfully using ionotropic gelation method. Various combinations of the drug and polymers were helpful in designing these polymers. Mean particle size, percentage release, entrapment efficiency and drug release varied with increasing drug polymer ratio. The assessment of release kinetics revealed that the drug release from microcapsules followed a Higuchi Model and the mechanism of drug release from the microcapsules was both diffusion and dissolution controlled. The drug and the polymers were compatible with each other. Further, toxicity and bioavailability studies in humans needs to be investigated in future studies in order to warrant the novel formulation design of Gliclazide microcapsules.

\section{ACKNOWLEDGEMENT}

The authors acknowledge Stand Pharma Lahore, Pakistan for their gift sample of Gliclazide used in this work. The authors are also grateful to Dr. Shahzad Naseem, Director solid state physics department, University of Punjab, Lahore, Pakistan for SEM studies. The necessary facilities provided by Department of Pharmacy, University of Sargodha for conducting the research are highly appreciated.

\section{REFERENCES}

Aulton EM. Pharmaceutics-The Science of Dosage Form Design. ELBS/Churchill Livingstone, London (1990).
Banker GS, Anderson NR. Tablets. In: Lachman L, Lieberman HA, Kanig JL. The Theory and Practice of Industrial Pharmacy. 2nd Ed. Philadelphia, PA: Lea \& Febiger; 1987.

Caro JJ, Getsios D, Caro I, Klittich WS, O'Brien JA. Economic evaluation of therapeutic interventions to prevent type 2 Diabetes in Canada. Diabetic Med. 2004; 21(11): 1229-1236.

George M, Abraham TE. Polyionic hydrocolloids for the intestinal delivery of protein drugs: Alginate and chitosan - A review. J Control Rel. 2006; 114(1):114.

Grbic S, Parojcic J, Ibric S, Djuric Z. In Vitro and In Vivo Correlation for Gliclazide Immediate-Release Tablets Based on Mechanistic Absorption Simulation. AAPS Pharm Sci Techol. 2011; 12(1):165-171.

Kobayashi K, Kimura M, Sakoguchi T, Hase A, Matsuoka A, Kaneko S. Pharmacokinetics of gliclazide in healthy and diabetic subjects. J Pharm Sci. 1984; 73(12): 1684-1687.

Kumar MS, Ramakrishna R, Kumar NN. Design and Development of Oral Controlled Release Formulations of Gliclazide Using Natural Polymers. Int Res J Pharm. 2010; 1(1): 233-242.

Logannathan V, Senthil Kumar K, Siva Prasada Reddy MV, Sreekanth N, Senthil Kumar B. Studies on preformulation compatibility between lomefloxacin and tablet excipients through DSC and X-ray diffraction analysis. Int J Pharm Excip. 2003; 38-49.

Nerup J, Platz P, Ortved AO, Christy M, Lyngsøe J, Poulsen JE, et al. Hl-A Antigens And Diabetes Mellitus. The Lancet.1974; 304(7885): 864-866.

Rahman Z, Kohli, Khar RK, Ali M, Charoo NA, Shamsher AAA. Characterization of 5-fluorouracil microspheres for colonic delivery. AAPS Pharm Sci Technol. 2006; 7(2): 113-121.

Ranjha NM, Khan H, Naseem S. Encapsulation and characterization of controlled release flursbiprofen loaded microspheres using beeswax as an encapsulating agent. J Mater Sci Mater Med. 2010; 21(5):1621-1630. 
Shariff A, Manna PK, Paranjothy KLK, Manjula M. Entrapement of andrographolide in crosss linked alginate pellets physico chemical characterization to study the pelletization of andrographolide. Pak $J$ Pharm Sci. 2007; 20(1): 1-9.

Singh MN, Hemant KSY, Ram M, Shivakumar HG. Microencapsulation: A promising technique for controlled drug delivery. Res Pharm Sci. 2010; 5(2): 65-77.

Tayade PT, Kale RD. Encapsulation of water insoluble drug by a cross linking technique: effect of process and formulation variables on encapsulation efficiency, particle size and in vitro dissolution rate. AAPS Pharm Sci Technol. 2004; 6(1): 112-119.
Vijaya DR, Razzak M, Tucker IG, Medlicott NJ. Encapsulation of vitamin B12 into poly lactide \& caprolactone microcapsules using $\mathrm{w}-\mathrm{O}-\mathrm{W}$ emulsion system. Indian Drugs. 2006; 43: 457-461.

Yamuda T, Onishi H, Machida Y. Sustained release kitoprofen micropartieles with ethyl cellulose and carboxymethyl ethyl cellulose. J Control Rel. 2001; 10-75(3): 271-282. 